\title{
ARTICLE OPEN AFF4 regulates osteogenic differentiation of human dental follicle cells
}

\author{
Qingyue Xiao ${ }^{1}$, Yuning Zhang ${ }^{1}$, Xingying $\mathrm{Qi}^{1}$, Yaqian Chen ${ }^{1}$, Rui Sheng ${ }^{1}$, Ruoshi $\mathrm{Xu}^{2}$, Quan Yuan ${ }^{3}$ and $\mathrm{Chenchen} \mathrm{Zhou}^{4}$
}

As a member of the AFF (AF4/FMR2) family, AFF4 is a transcription elongation factor that is a component of the super elongation complex. AFF4 serves as a scaffolding protein that connects transcription factors and promotes gene transcription through elongation and chromatin remodelling. Here, we investigated the effect of AFF4 on human dental follicle cells (DFCs) in osteogenic differentiation. In this study, we found that small interfering RNA-mediated depletion of AFF4 resulted in decreased alkaline phosphatase (ALP) activity and impaired mineralization. In addition, the expression of osteogenic-related genes (DLX5, SP7, RUNX2 and $B G L A P$ ) was significantly downregulated. In contrast, lentivirus-mediated overexpression of AFF4 significantly enhanced the osteogenic potential of human DFCs. Mechanistically, we found that both the mRNA and protein levels of ALKBH1, a critical regulator of epigenetics, changed in accordance with AFF4 expression levels. Overexpression of ALKBH1 in AFF4-depleted DFCs partially rescued the impairment of osteogenic differentiation. Our data indicated that AFF4 promoted the osteogenic differentiation of DFCs by upregulating the transcription of $A L K B H 1$.

\section{INTRODUCTION}

The integrity of dentition is of significant importance to oral health. Dentition defects can have adverse influences on oral functions, such as the vocalization, masticatory function and aesthetics, thus diminishing the patients' quality of life. ${ }^{1}$ There is a high prevalence of periodontal problems, but tooth loss is not a good option. As a chronic infectious disease, periodontal disease is characterized by the inflammation of supporting tissues, such as gingiva, the periodontal membrane, the cementum and alveolar bone, resulting in loose or lost teeth. ${ }^{2}$ Dental prosthetic treatment plays a vital role in achieving mechanical and partial functional restoration. However, the ultimate goal of treatment is regenerating the damaged periodontal tissue to restore the original composition and function. ${ }^{3}$ With this goal in mind, periodontal tissue regeneration engineering provides a potential solution. The oral cavity contains rich sources of mesenchymal stem cells, and previous studies have shown that these stem cells have a superior ability to regenerate tissue in vitro, especially in terms of osteogenesis. ${ }^{4}$ Dental follicle cells (DFCs), which show excellent osteogenesis potential, can hopefully be a substitute for traditional marrow stem cells. ${ }^{5}$

The dental follicle, originating from the ectodermal mesenchyme, is a loose connective tissue that wraps around the developing tooth germ. During tooth development, periodontal supporting tissues originate from dental follicles and play a role in buffering, supporting, remodelling and regenerating the developing teeth. ${ }^{6}$ Furthermore, periodontal supporting tissues embody distinct embryonic features, such as high self-renewal capability, prominent proliferation potential, pluripotency, heterogeneity and multidirectional differentiation. ' The human dental follicle harbours a mass of stem cells that have a dramatic effect on periodontal tissue development and bone remodelling. DFCs can differentiate into osteoblasts, fibroblasts, neurons and adipocytes under the corresponding induction in vitro. ${ }^{8}$ DFCs possess several excellent characteristics: easy acquisition, minimal surgical trauma, excellent cell fusion ability and the ability to be stored a long time. Given their unique properties, DFCs have been regarded as promising seed cells for use in periodontal tissue regeneration engineering. ${ }^{9}$ Therefore, it is of great significance to explore the core target genes and regulatory mechanisms involved in periodontal tissue development, hopefully providing insights into strategies for periodontal tissue engineering. ${ }^{10,11}$

The differentiation of stem cells is a highly regulated process influenced by numerous factors at each step. As a critical step in gene expression, transcription is precisely regulated. ${ }^{12}$ As a central enzyme, RNA Polymerase II (RNA Pol II) is responsible for producing noncoding RNAs as well as pre-mRNAs. ${ }^{13}$ Mediated by RNA Pol II, gene transcription is a strictly controlled procedure that has four phases: transcription initiation, transcription pausing, transcriptional elongation and transcriptional termination. Among these processes, elongation is a significant rate-limiting stage that dictates the expression of the entire genome. ${ }^{14}$

The transcriptional elongation complex is a regulatory intermediate in the transcriptional elongation stage. ${ }^{15}$ After binding to the RNA polymerase, it transforms into a special spatial conformation that interacts with RNA, DNA and other elongation

\footnotetext{
${ }^{1}$ State Key Laboratory of Oral Diseases \& National Clinical Research Center for Oral Diseases \& West China Hospital of Stomatology, Sichuan University, Chengdu, China; ${ }^{2}$ State Key Laboratory of Oral Diseases \& National Clinical Research Center for Oral Diseases \& Department of Cariology and Endodontology, West China Hospital of Stomatology, Sichuan University, Chengdu, China; ${ }^{3}$ State Key Laboratory of Oral Diseases \& National Clinical Research Center for Oral Diseases \& Department of Oral Implantology, West China Hospital

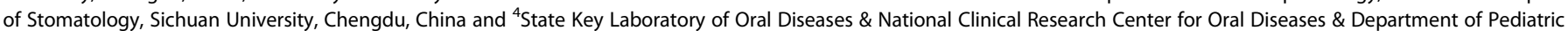
Dentistry, West China Hospital of Stomatology, Sichuan University, Chengdu, China

Correspondence: Quan Yuan (yuanquan@scu.edu.cn) or Chenchen Zhou (zhouchenchen5510@163.com)
}

Received: 31 December 2019 Revised: 21 February 2020 Accepted: 23 February 2020

Published online: 30 June 2020 


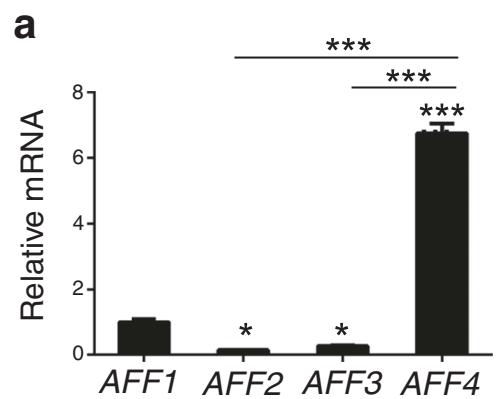

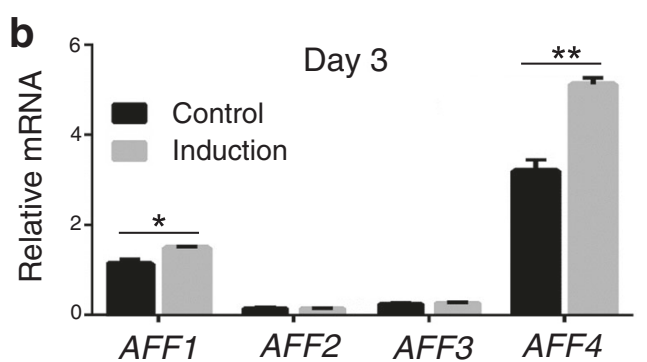

C

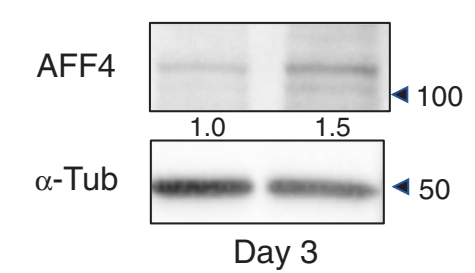

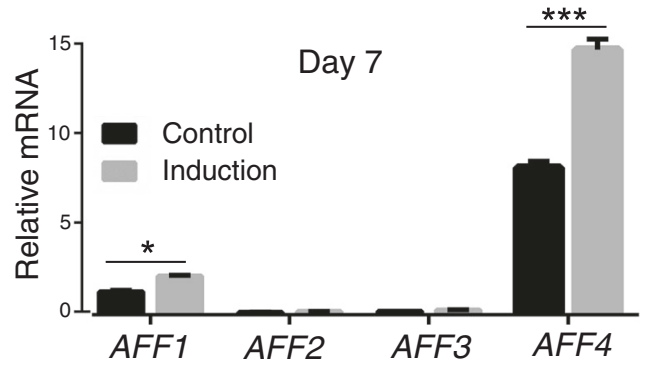

Control Induction

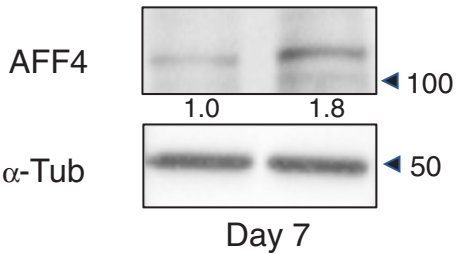

Fig. 1 Expression of AFF4 in DFCs. a Quantitative RT-PCR shows that the expression of AFF4 is the highest among AFF family members. $n=3$. ${ }^{* * *} P<0.001$, and ${ }^{*} P<0.05$. b Quantitative RT-PCR of AFF family members after osteogenic differentiation induction for 3 and 7 days. $n=3$. ${ }^{* * *} P<0.001,{ }^{* *} P<0.01$, and ${ }^{*} P<0.05$. c Western blot analyses of AFF4 after osteogenic differentiation induction for 3 and 7 days. The quantitation of the WB is shown

factors, completing signal reception and influencing transcription decisions during transcription pausing. ${ }^{16}$ With more research on the transcriptional elongation process, the super elongation complex (SEC) is receiving increasing attention for its communication with multitudinous target genes during transcriptional elongation, which is regarded as a significant rate-limiting stage. ${ }^{17}$ The SEC is promptly recruited to the target genes in response to differentiation signals, where it then releases the paused RNA Pol II to enable dynamic transcription. ${ }^{18}$ It has a positive regulating effect on transcriptional elongation, activating transcriptional activity and improving elongation efficiency. As a variable multiprotein complex, the SEC consists of several subunits: positive elongation factor $b$ (P-TEFb), an AFF protein family member (scaffold protein), an ELL protein family member (the elongation factor of RNA Pol II) and a partner protein AF9 or ENL. ${ }^{19}$

Localized in the nucleus, the AFF protein family comprises four members: AFF $1 / 2 / 3 / 4 .^{20}$ In the SEC, significant emphasis is placed on AFF1 or AFF4, which serve as scaffold proteins that promote the assembly of other subunits and maintain the stability of the complex. ${ }^{19,21}$ AFF1 and AFF4 have been extensively implicated in the control of HIV transcription. At the RNA level, a previous analysis demonstrated that the effect of AFF4 is stronger than that of AFF1 on early HIV transcription. ${ }^{22}$ Furthermore, AFF1 and AFF4 are both critical epigenetic regulators, but they have different roles during the osteogenic differentiation of human mesenchymal stem cells (MSCs). Previous results show that AFF4 plays a pivotal role in stimulating MSC osteogenic differentiation. ${ }^{23}$

In this study, we tested the impact of AFF4 on the osteogenic differentiation of DFCs, and we analysed the underlying regulatory mechanism. Our results show that AFF4 can stimulate the osteogenic differentiation of DFCs by regulating $A L K B H 1$ expression.

\section{RESULTS}

Expression of AFF4 in DFCs

First, we investigated the expression of AFF4 in DFCs. As shown by quantitative RT-PCR (qRT-PCR) analysis, among the AFF family members, the expression of AFF4 was the highest. For other family members, AFF1 had the next highest expression, while the expression of AFF2 or AFF3 was low (Fig. 1a).

We also conducted osteogenic induction on DFCs and analysed dynamic changes in expression of AFF family members. The results of qRT-PCR indicated that the expression of AFF4 dramatically increased after induction, while there was no significant difference in AFF2 or AFF3. (Fig. 1b). The analyses of western blot and semiquantification of AFF4 confirmed its higher expression after osteogenic induction relative to its levels before induction (Fig. 1C), suggesting that during the osteogenic differentiation of DFCs, AFF4 is a potential regulator.

Depletion of AFF4 decreases osteogenic differentiation of DFCs To illustrate the importance of AFF4 in the osteogenic differentiation of DFCs, we knocked down AFF4 in DFCs by treatment with a small interfering RNA (siRNA). Two days after transfection, qRT-PCR and western blot analyses were used to test the knockdown efficiency (Fig. 2a, b). We found that the staining of alkaline phosphatase (ALP), an essential marker of early osteogenic 
a

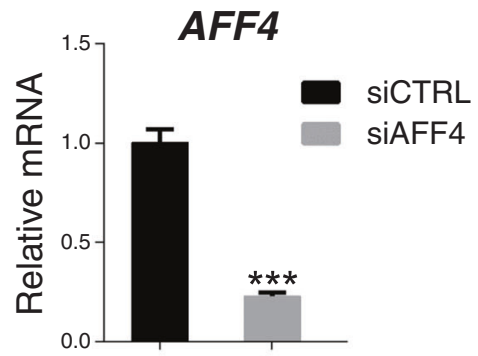

C

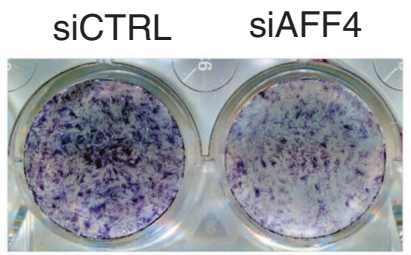

Day 7

e SICTRL SIAFF4

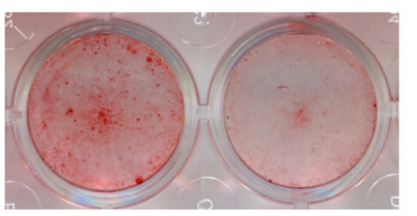

Day 21

g

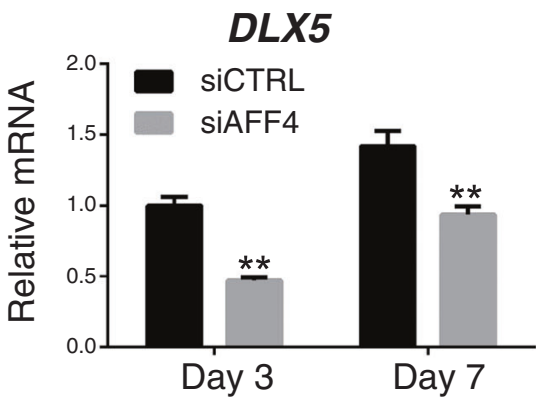

i

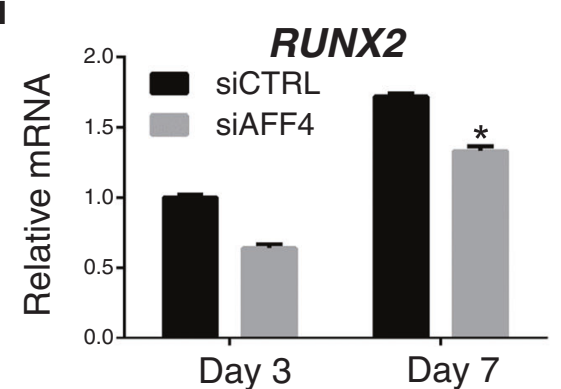

b SICTRL SIAFF4

AFF4

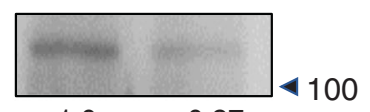

$\alpha-$ Tub

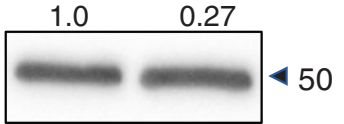

d

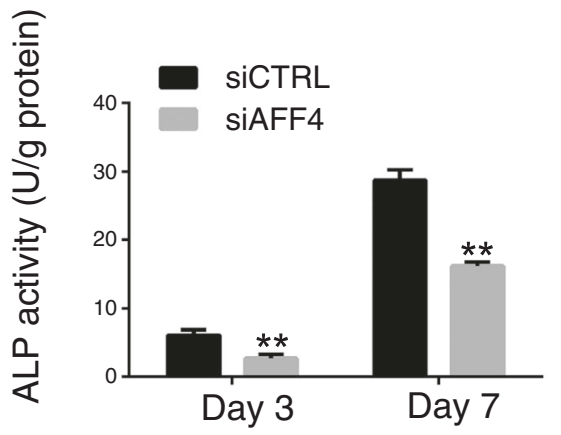

f

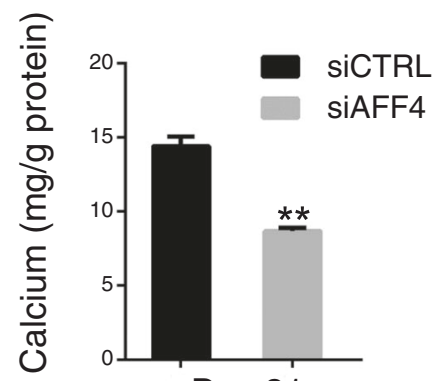

Day 21

h

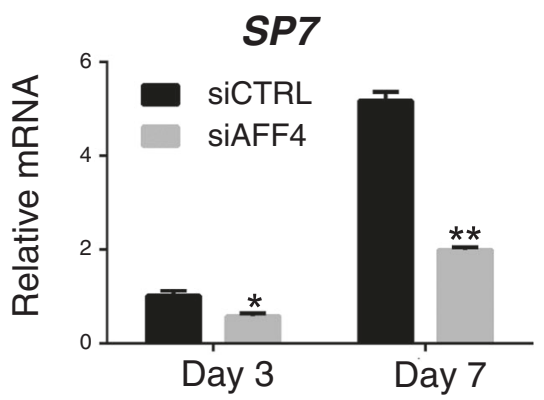

j

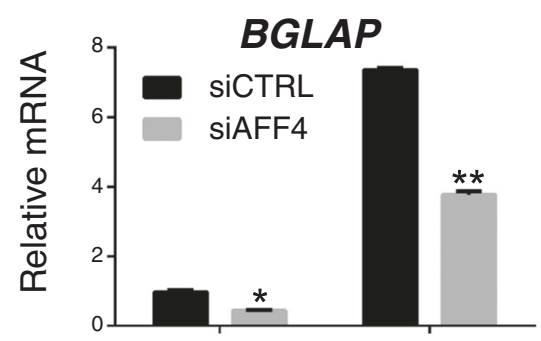

Day 3

Day 7

Fig. 2 Depletion of AFF4 decreases osteogenic differentiation of DFCs. a Quantitative RT-PCR showing the successful knockdown of $A F F 4$. $n=3$. ${ }^{* * *} P<0.001$. b Western blot analysis and semiquantification of AFF4. $\mathbf{c}$ Representative ALP staining images of DFCs. $\mathbf{d}$ Quantitative assessment of ALP activity. $n=5 .{ }^{* *} P<0.01$. e Representative images of ARS staining. $\mathbf{f}$ Quantitative assessment of calcium mineralization. $n=5 .{ }^{* *} P<0.01$. g-j Quantitative RT-PCR demonstrating the reduced expression of DLX5, SP7 and BGLAP. $n=3 .{ }^{*} P<0.05$, and ${ }^{* *} P<0.01$ 
differentiation, was significantly reduced in the siRNA-mediated AFF4 depletion group after osteogenic induction for 7 days (Fig. 2c). The results of quantitative ALP activity measurements also confirmed previous observations after osteogenic induction for 3 days and 7 days (Fig. 2d). Alizarin red S (ARS) staining and calcium deposition analysis were performed to investigate the impact of AFF4 on extracellular matrix mineralization. The results revealed that there was less ARS staining after AFF4 depletion than there was in control cells (Fig. 2e). A quantitative measurement of calcium concentration, showed that it was also decreased as AFF4 was depleted (Fig. 2f), indicating that AFF4 was significant in the mineralization process. In addition, we evaluated the expression of $D L X 5, S P 7, R U N X 2$ and BGLAP, and we found that these osteogenicrelated genes were repressed in AFF4-depleted cells relative to their levels in control cells (Fig. $2 g-j$ ).

\section{Overexpression of AFF4 promotes osteogenic differentiation of DFCs}

We wondered whether the overexpression of AFF4 could have an opposite effect to enhance osteogenic differentiation. DFCs were stably infected with lentivirus expressing GFP or AFF4. qRT-PCR and western blot analyses were performed to verify the overexpression efficiency (Fig. 3a, b). AFF4 overexpression caused increased ALP staining and ALP activity after osteogenic induction (Fig. 3c, d). In addition, ARS staining and extracellular matrix mineralization were also markedly enhanced over that of control cells (Fig. 3e, f). Moreover, the overexpression of AFF4 stimulated the expression of DLX5, SP7 and BGLAP, as shown by qRT-PCR on osteogenic induction at day 3 and day 7 (Fig. $3 g-j$ ).

AFF4 regulates the expression of ALKBH1

ALKBH1 is a critical epigenetic factor in the osteogenic differentiation of $\mathrm{MSCs}^{24}$ Therefore, we wondered whether AFF4 could regulate the transcription of $A L K B H 1$. First, we found that the expression of ALKBH1 was significantly reduced after AFF4 depletion at both the mRNA and protein levels (Fig. 4a, b). Conversely, the overexpression of AFF4 induced the expression of ALKBH1 (Fig. 4c, d). Next, an anti-AFF4 ChIP assay was conducted to further reveal the relationship between AFF4 and ALKBH1. We showed that AFF4 bound to the promoter region of $A L K B H 1$ and that the ChIP signal was largely abolished by AFF4 depletion (Fig. 4e).

Overexpression of $A L K B H 1$ partially rescues osteogenic differentiation of AFF4-deficient DFCs

To further elucidate the regulatory mechanism involving $A L K B H 1$, rescue experiments were conducted by using a lentivirus to overexpress ALKBH1 in AFF4-depleted DFCs. ALKBH1 overexpression was confirmed to be efficient (Fig. 5a, b). Overexpression of $A L K B H 1$ in DFCs rescued the suppressed ALP staining and ARS staining caused by AFF4 depletion (Fig. 5c, d). Quantitative measurement of ALP activity and of calcium concentrations also showed that overexpression of $A L K B H 1$ significantly abolished the inhibition of osteogenic differentiation caused by AFF4 depletion (Fig. 5e, f). Finally, we performed qRT-PCR experiments, which revealed that $A L K B H 1$ overexpression restored the inhibited mRNA expression of DLX5, SP7, RUNX2 and BGLAP (Fig. 6a-d).

\section{DISCUSSION}

Periodontal tissue regeneration is a key part of the treatment of periodontal disease. ${ }^{3}$ Pathological alveolar resorption is based on the negative balance of bone reconstruction with the decline of osteoblast function and the increase of osteoclast activity, which has an impact on the reconstruction of alveolar bone. ${ }^{25}$ The osteogenic differentiation of dental stem cells is a complicated activity, which follows the differentiation sequence of producing first osteogenesis progenitor cells, osteogenesis precursor cells and osteoblasts that eventually differentiate into bone cells. This process involves various types of intracellular and intercellular signal transmission and pathways, which constitute a complex signal transduction network. ${ }^{26}$

DFCs have the extraordinary ability to differentiate into different and diverse cell types. The periodontal tissues, such as the cementum, periodontal ligament and alveolar bone, all originate from dental follicles. The dental sac surrounding the tooth germ is the matrix where DFCs reside. DFCs, with the unique properties of stem cells, are important for the growth of tooth roots and tooth eruption. Various periodontal progenitor cells originate from the dental follicle and are associated with the construction of periodontal tissue. ${ }^{8,12}$

Dental follicle cells can differentiate into multiple cell types, forming periodontal ligament, cementum and alveolar bone during the development of periodontal tissue. ${ }^{27}$ A previous study showed that DFCs can rescue the degeneration of inflamed periodontal ligament stem cells, suggesting a promising future in periodontal regeneration. ${ }^{7}$ In addition, DFCs can form root-like tissues upon being transferred into a treated dentin matrix scaffold in the alveolar fossa. ${ }^{5}$ DFCs have multiple differentiation competencies, they have low immunogenicity and are easily accessed. Given their remarkable properties, DFCs have been considered pivotal seed cells in periodontal tissue engineering and hopefully provide a novel approach to regeneration therapy. ${ }^{7,28}$

Previous studies have emphasized the accurate transfer of genetic information, which is essential for life. RNA Pol II can accelerate translation by the enzymatic machinery to transcribe protein-coding genes into mRNA. ${ }^{29}$ Transcriptional elongation is a vital step during gene transcription, and the SEC has a positive regulatory effect on this process. ${ }^{22}$

For the initial induction stage of transcription, an external stimulus is required. In mammals, the different SECs form in different combinations with common activators to regulate different genomes. SECs perform initiation and extension functions in gene transcription and can also modify the transcriptional region via histone methylation. ${ }^{30}$ The SEC is necessary in the stage of Pol II release from the proximal suspension of the gene promoter and transcription of HIV-1 gene activation. ${ }^{22,31}$ The recruitment of the SEC by genomes can occur specifically or nonspecifically. Specific recruitment means that the SEC can be recruited to certain sites within the genome as directed by the DNA binding domain, and a particular recruitment factor can stabilize the structure of the SEC. Detection of non-specific recruitment at the genome-wide level shows that SEC binding is widespread in the genome, suggesting that the SEC also plays a corresponding role in the basic transcription process and that the mechanism might be related to transcriptional coactivators. ${ }^{19,32}$

The SEC is usually compromised of four subunits: a member from each of the AFF and ELL protein families, P-TEFb, and AF9 or ENL. The P-TEFb complex is a heterodimer that consists of CDK9 and CyclinT. It is essential for eukaryotic transcription and is necessary for most gene expression; its regulatory mechanism was first elucidated in HIV transcription. Moreover, Ser-2 and Thr-4 of the CTD, a heptapeptide structure, are phosphorylated by P-TEFb during transcription, providing the necessary conditions for transcriptional elongation. ${ }^{22,33}$ ELL2 is a functional transcription elongation factor that is able to accelerate the catalytic rate of transcription. RNA Pol II inhibition is prevented by the withdrawal of RNA Pol II from the $3^{\prime}$ end domain in the newly synthesized RNA on account of the activity of ELL2. At the same time, the half-life of ELL2 protein is remarkably improved after binding to AFF1 or AFF4, which can protect ELL2 from degradation. ${ }^{34}$ Moreover, demonstrating high homology, ENL and AF9 can help to achieve attachment between SEC and RNA Pol II by interacting with the RNA Pol II and becoming linked to the PAF1 complex (PAF1c). In addition, the YEATS region of ENL and AF9, a novel acetyl-binding 
a

AFF4

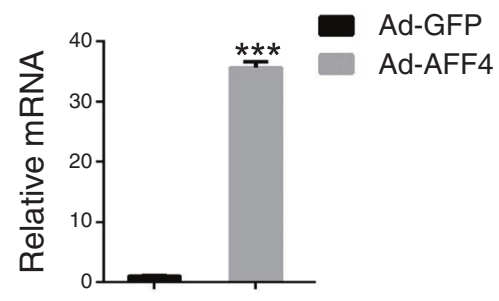

C

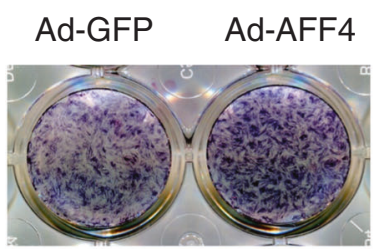

Day 7

e

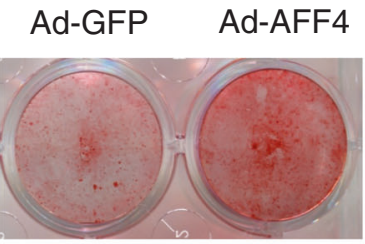

Day 21

g

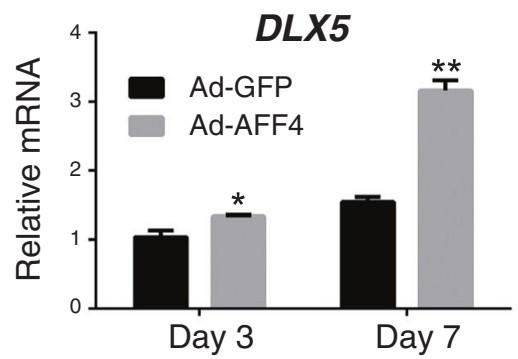

i

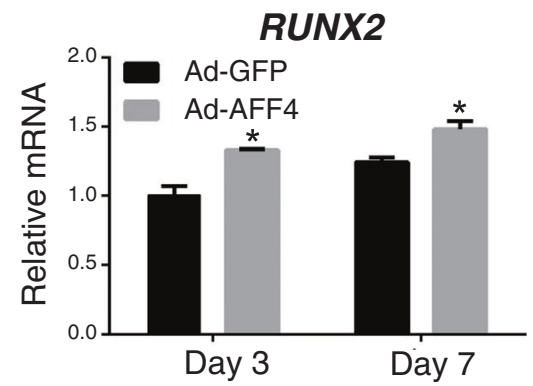

b

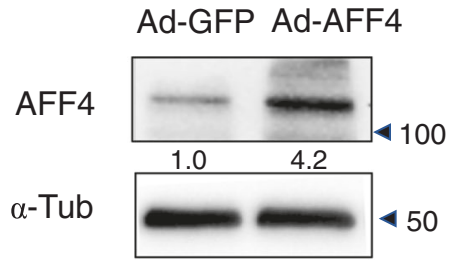

d

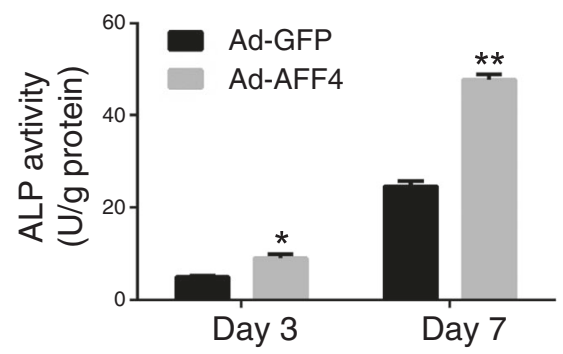

f

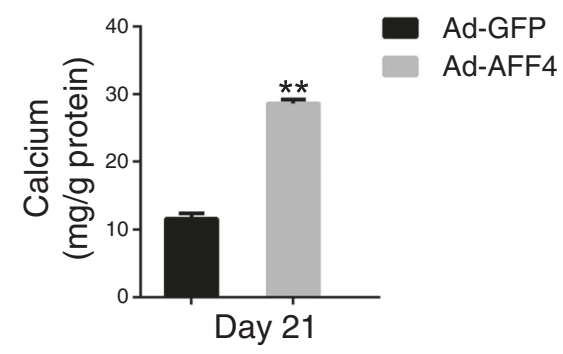

h

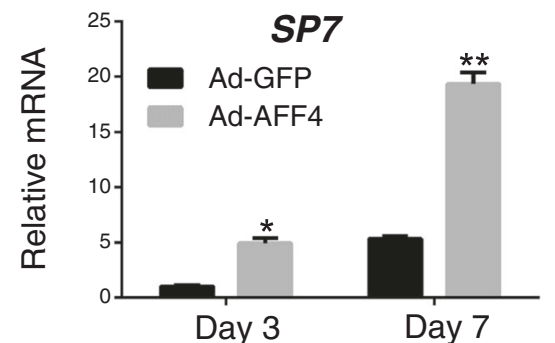

j

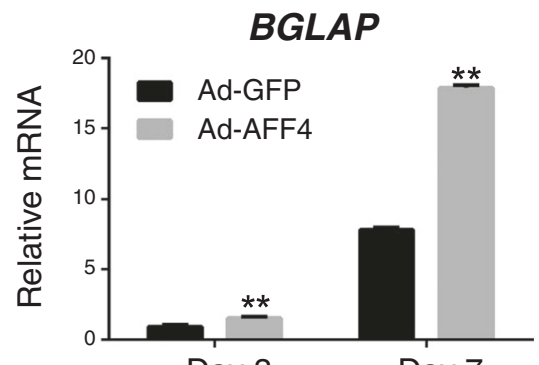

Fig. 3 Overexpression of AFF4 promotes osteogenic differentiation of DFCs. a Quantitative RT-PCR shows that the mRNA level of AFF4 is significantly increased. $n=3$. ${ }^{* * P}<0.001$. $\mathbf{b}$ Western blot analysis and semiquantification of AFF4. c Representative ALP staining images of DFCs. Increased expression of AFF4 enhances the intensity of the staining. d Quantitative ALP activity assessment. $n=5$. ${ }^{* * * P} P<0.001$. e Representative ARS staining images of DFCs. f Quantitative calcium mineralization assessment. $n=5$. ${ }^{* *} P<0.01$. g-j Quantitative RT-PCR displays the increased mRNA expression of $D L X, S P 7$ and BGLAP. $n=3$. ${ }^{*} P<0.05$ and ${ }^{* *} P<0.01$ 

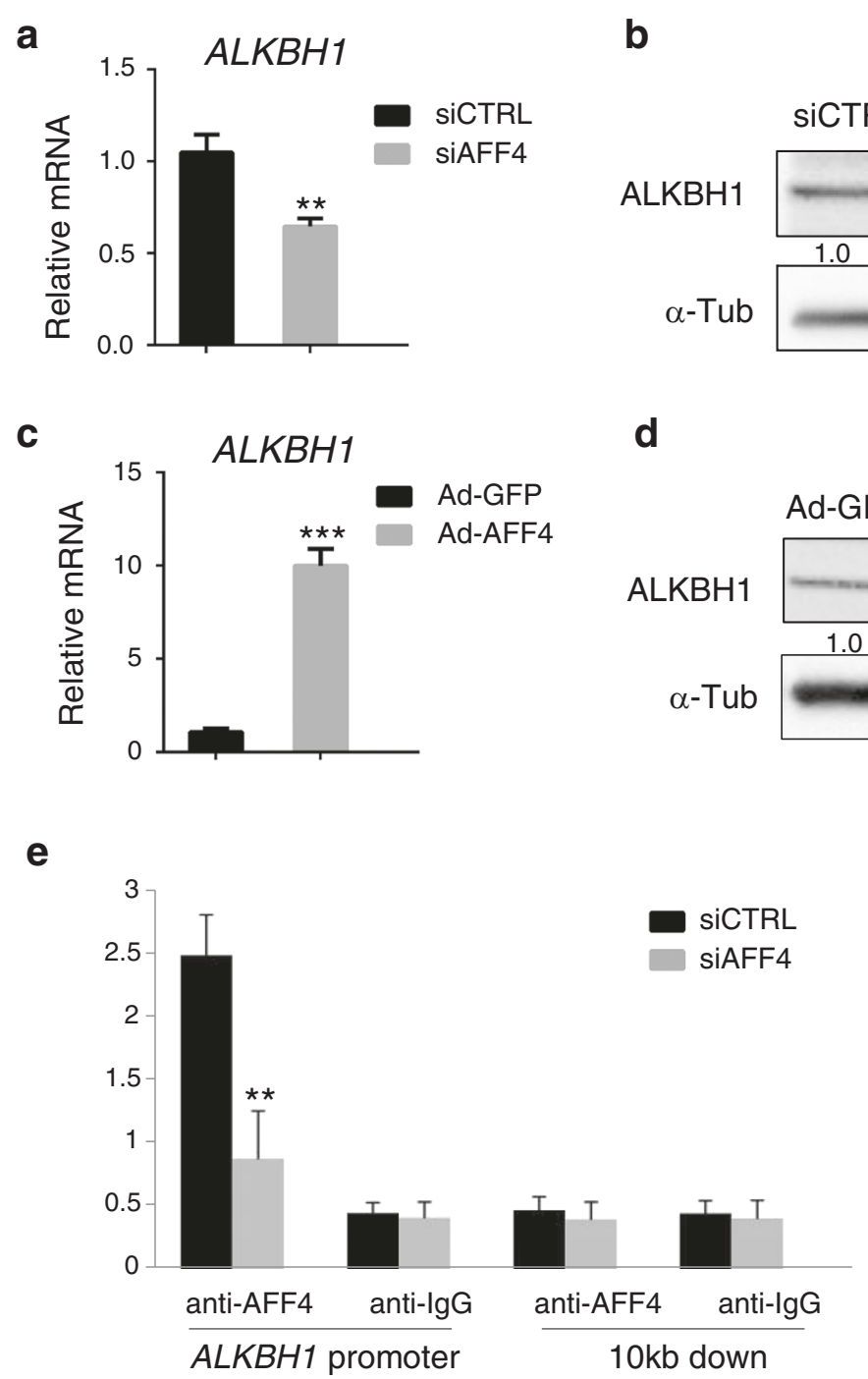

Fig. 4 AFF4 regulates the expression of ALKBH1. $\mathbf{a}$, b Quantitative RT-PCR, western blot and quantification of ALKBH1 after the depletion of AFF4. $n=3$. ${ }^{* *} P<0.01$. c, d Quantitative RT-PCR, western blot and quantification of ALKBH1 after AFF4 overexpression. $n=3$. ${ }^{* * * P}<0.001$. e ChIP assay for AFF4 demonstrates that it binds to the promoter region of $A L K B H 1 . n=4 .{ }^{* *} P<0.01$

module that is directly associated with histone acetylation and H3K79 methylation, is an indispensable structure involved in enhancing transcriptional stimulation. ${ }^{35,36}$

Formation of an SEC with AFF1 or AFF4 is a primary control factor in cancer pathogenesis and development. SEC-like 2 (SECL2) or SEC-like 3 (SEC-L3), containing factor AFF2 or AFF3, respectively, was also discovered through biochemical isolation. Among the SEC family members, AFF4-formed SECs play a primary role in cells after receiving rapid transcriptional induction and demonstrates high kinase activity of the polymerase II C-terminal domain in cells. ${ }^{32,34,37}$ Thus, SECs have complex combination modes with diverse internal variability. SECs, as a large family system, may have different mechanisms driving their regulation of gene transcription, which need further exploration.

By promoting the process of RNA elongation, the AFF family of proteins serve as transcriptional activators, and the family consists of four members. ${ }^{20}$ AFF1 and AFF4 originate from the same branch in the phylogenetic trees, while AFF2 and AFF3 belong to another branch. AFF1 and AFF4 are both known scaffold proteins in SECs, but they exist in different SECs, demonstrating primarily distinguishable gene target specificities. A previous study revealed that both AFF1 and AFF4 can efficiently activate HIV gene transcription, and the results of these functions were confirmed by b

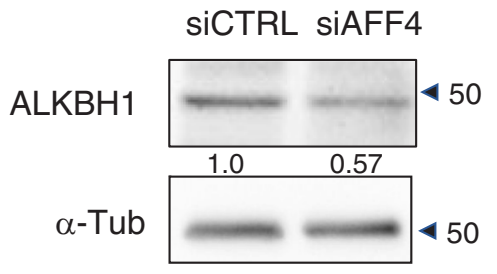

d

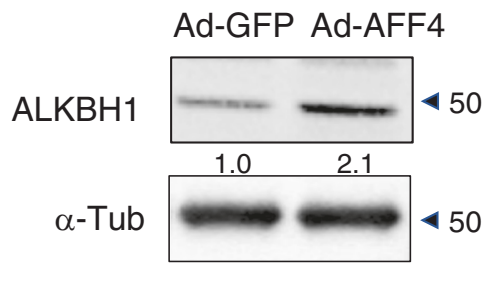

analysis of the transcriptional synthesis efficiency of the SECs at the mRNA level. In detail, AFF1-formed SECs are more efficient when functioning the viral protein Tat, while AFF4-formed SECs are more potent upon heat shock under the induction of HSP70. The difference in their ability to activate early HIV transcription results from the different recruitment of viral promoters and their ability to interact with PAFc. ${ }^{31}$

AFF1 is a fusion partner that is strongly expressed in mixedlineage leukaemia (MLL) and acute lymphoblastic leukaemia $(\mathrm{ALL})^{38}$ AFF2, as an X-linked gene, and it has considerable expression in the placenta and brain. The repressed expression of AFF2 can result in intellectual disability and mental retardation, such as FRAXE. ${ }^{39}$ AFF3 is also involved in fusion activity with intellectual disability. The expression of AFF3 is high in lymphocytes, and it is upregulated in cortical neurons relative to other cell types. ${ }^{40}$

AFF4 contains a common conserved C-terminal homology domain that is also found in the rest of the AFF protein members, and it has an $\mathrm{N}$-terminal region that interacts with other subunits of the SEC. Among its family members, AFF4 is the most widely distributed factor in human tissues, and it is associated with a variety of life activities. ${ }^{32}$ As the fused subunit of MLL chimaeras, AFF4 can bring transcription elongation factors to targeted 
a

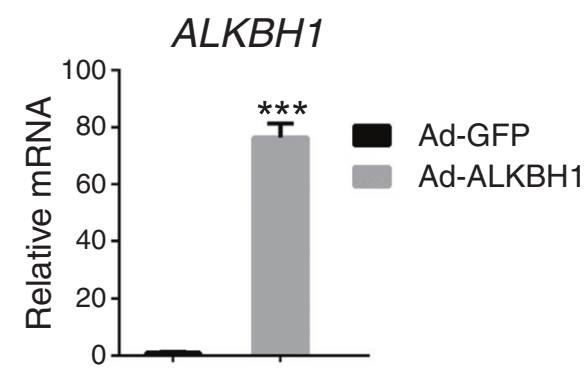

C

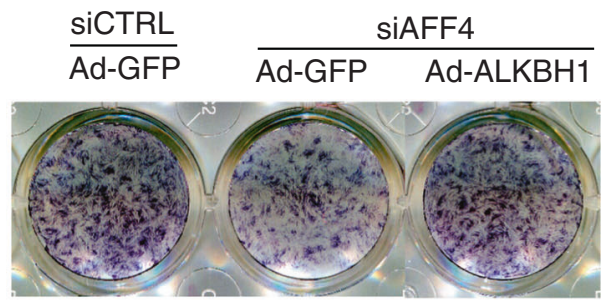

Day 7

e

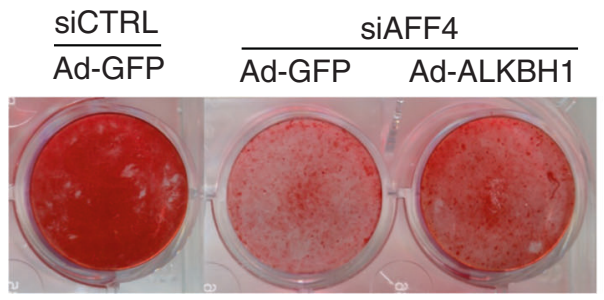

Day 21 b

$$
\frac{\text { siCTRL }}{\text { Ad-GFP }} \frac{\text { siAFF4 }}{\text { Ad-GFP Ad-ALKBH1 }}
$$

Flag
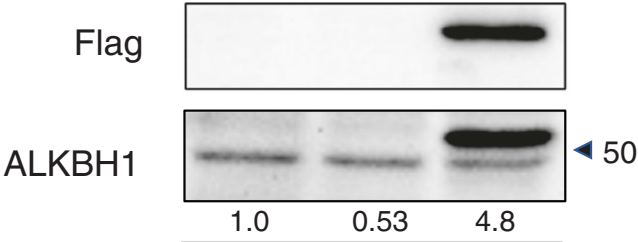

AFF4

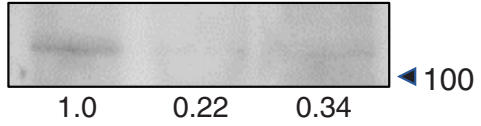

$\alpha$-Tub

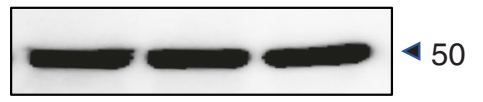

d

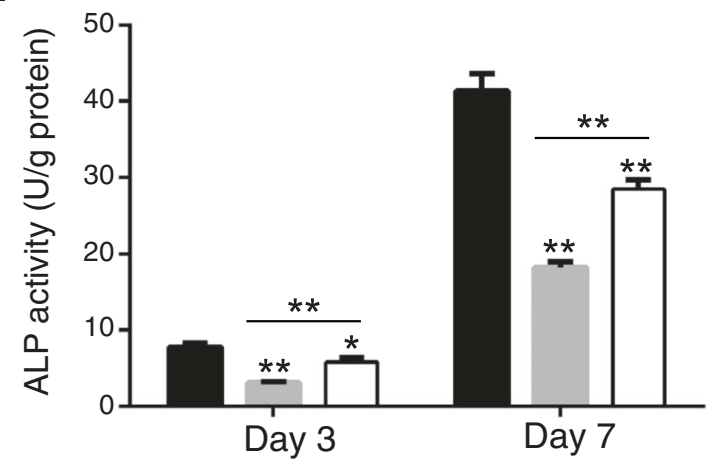

f

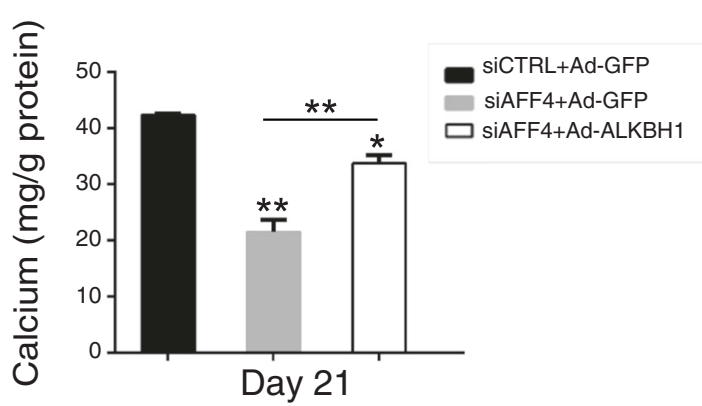

Fig. 5 Overexpression of $A L K B H 1$ partially rescues osteogenic differentiation of $A F F 4$-deficient DFCs. a The mRNA level of $A L K B H 1 . n=3$. ${ }^{* *} P<$ 0.001. b Western blot analyses of ALKBH1 and AFF4. Semi-quantifications of WB are shown. c, d Representative images of ALP staining and quantification of ALP activity. $n=5$. ${ }^{*} P<0.05$, and ${ }^{*} P<0.01$. e, $\mathbf{f}$ Representative images of ARS staining and quantitative analyses of calcium mineralization. $n=5$. ${ }^{*} P<0.05$, and ${ }^{*} P<0.01$

leukaemic genes. In leukaemic cells, AFF4-depleted SEC can result in an obvious reduction in MLL chimaera target gene expression. ${ }^{20}$ AFF4 can activate HIV-1 proviral transcription and transcription elongation factors, which are dispersed along a flexible AFF4 scaffold and can be recruited by the HIV-1 protein Tat. ${ }^{41}$ In head and neck squamous cell carcinoma cells, AFF4 can improve tumour initiation and tumorigenesis capability though SOX2. ${ }^{42}$ Previous research found that Aff4/Af5q31-null mice exhibited azoospermia, suggesting a nonnegligible effect on Sertoli cells. Moreover, in regard to FRAXE, AFF4 remains functionally redundant during brain development.

To explore the effect of AFF4 on osteogenic differentiation of human DFCs, we first investigated its expression level and found that the expression of AFF4 was the highest among the members of the AFF family. Then, the knockdown and overexpression of AFF4 were achieved through siRNA and lentivirus treatment, respectively. AFF4 depletion repressed the osteogenic differentiation ability of DFCs. Accordingly, the overexpression of AFF4, mediated by lentiviral treatment, promoted osteogenic differentiation.

For stem cells, differentiation into specialized cells requires specific cell signals and epigenetic regulation. Epigenetic changes, such as DNA methylation and histone modifications and changes, mainly occur in association with noncoding RNA expression and deregulation of epigenetic processes. They can adjust gene expression levels in the multistep process of carcinogenesis. ${ }^{43}$ During the differentiation course of stem cells, epigenetic regulation points to functionally correlative modifications within the genome, while the nucleotide sequence remains unchanged. ${ }^{44}$

As a 2-oxoglutarate and $\mathrm{Fe}^{2+}$-dependent hydroxylase, ${ }^{45}$ ALKBH1 functions as a demethylase for a new epigenetic 

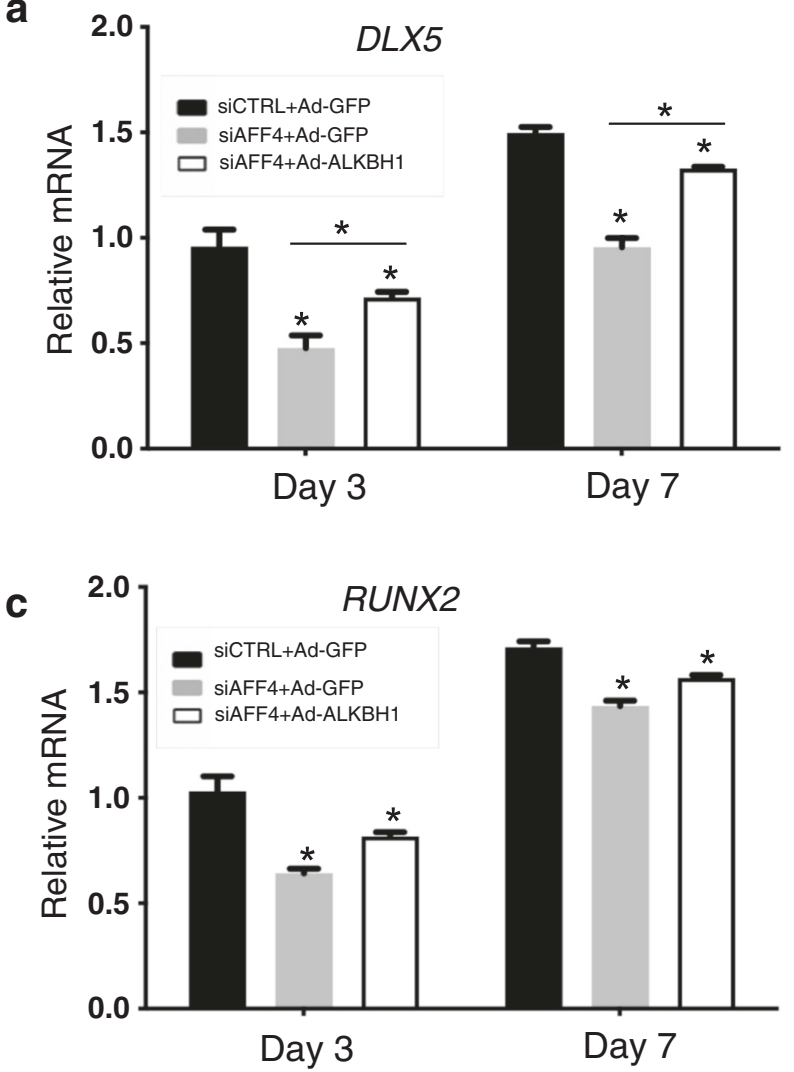

b

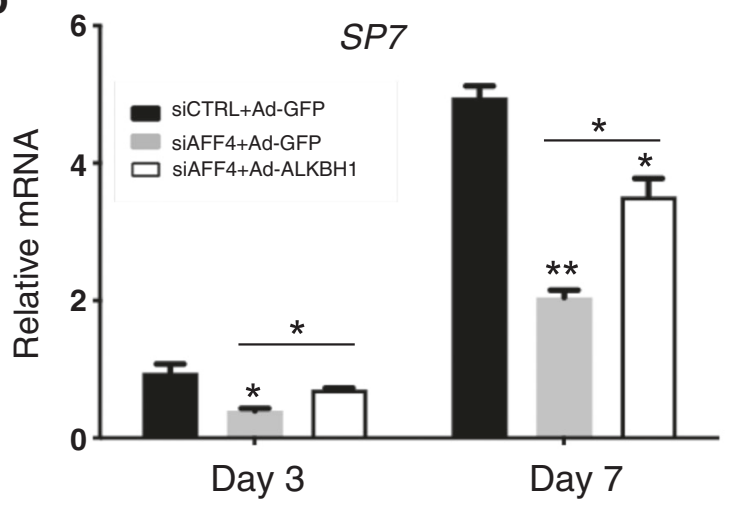

d

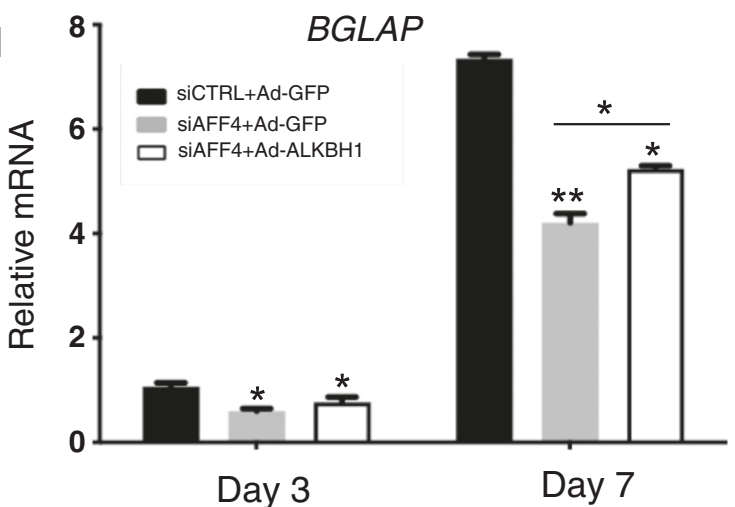

Fig. 6 The expression of osteogenic-related genes in ALKBH1-overexpressing DFCs. a-d Quantitative RT-PCR reveals that overexpression of $A L K B H 1$ restores the blunted expression of DLX, SP7, RUNX2 and BGLAP. $n=3$. ${ }^{*} P<0.05$, and ${ }^{* *} P<0.01$

modification of DNA $\mathrm{N}^{6}$-methyladenine $\left(\mathrm{N}^{6}-\mathrm{mA}\right)$, and provides dual effects regarding the central network of transcription factors regulating embryonic stem cells. ${ }^{46,47}$ ALKBH1 possesses high demethylation activity, indicating its effect on silencing epigenetic genes. ${ }^{48}$ It participates in damage reversal through oxidative demethylation of 1-methyladenine and 3-methylcytosine, and it is involved in the regulation of transcription mechanisms. ${ }^{49}$ ALKBH1 also has a prominent influence on epigenetic regulation by accommodating pluripotency markers and differentiation genes during embryogenesis. In embryonic stem cells, ALKBH1 is involved in the central network transcription factors regulating pluripotency. A deficiency in ALKBH1 delays the expression of pluripotency-related markers and postpones the induction of genes that participate in early differentiation. ${ }^{45}$ Our previous research also notes that ALKBH1 has a close relationship with the osteogenic differentiation of human MSCs and is well described as a factor in MSCs that promotes osteogenesis. ${ }^{24}$ Given these research facts, we hypothesized that $\mathrm{ALKBH} 1$ might be involved in the osteogenic differentiation mechanism of DFCs. Here, we found that ALKBH1 also functions as a stimulus for osteogenic differentiation of human DFCs under the regulation of AFF4.

In summary, our data indicate that AFF4 is highly expressed in DFCs. AFF4 depletion impairs the osteogenic differentiation of DFCs. In contrast, the lentivirus-mediated overexpression of AFF4 promotes osteogenic differentiation of DFCs. Mechanistically, AFF4 enhances $A L K B H 1$ transcription, producing a positive effect on the osteogenic differentiation of human DFCs. In this study, the results reveal that the scaffold protein AFF4, as a critical epigenetic regulator, plays an important role during the osteogenic differentiation of human DFCs. Our study on the molecular mechanism of AFF4 in regulating DFC osteogenic differentiation and its key role in determining the fate of stem cells can provide an important experimental basis for the clinical treatment of bone repair and reconstruction in periodontal diseases.

\section{MATERIALS AND METHODS}

Cell culture

Human DFCs were harvested from tooth buds of impacted third molars with undeveloped roots. Patients who underwent extractions for orthodontic reasons were 8-18 years old and had provided informed consent; there was no gender requirement. Procedures took place at the West China Hospital of Stomatology, Sichuan University. None of these selected patients had systemic disease, a history of radiotherapy, had undergone chemotherapy or had a history of smoking. All experimental procedures and protocols were approved by West China Hospital of Stomatology, Sichuan University.

Isolated follicle tissues were immediately transferred into phosphate-buffered saline (PBS) supplemented with 100 units $\mathrm{mL}^{-1}$ penicillin (Gibco) and $100 \mu \mathrm{g} \cdot \mathrm{mL}^{-1}$ streptomycin (Gibco) once obtained. The human dental follicles were cut into approximately $1 \mathrm{~mm}^{3}$ tissue blocks and digested for $1 \mathrm{~h}$ at $37^{\circ} \mathrm{C}$ with agitation in PBS containing $3 \mathrm{mg} \cdot \mathrm{mL}^{-1}$ type I collagenase (Gibco) and $3 \mathrm{mg} \cdot \mathrm{mL}^{-1}$ dispase (Gibco).

The digested suspensions were transferred into $21 \mathrm{~cm}^{2}$ culture dishes and were cultured in alpha-minimum Eagle's medium (aMEM) (Gibco) supplemented with $20 \%$ foetal bovine serum (FBS) (Gibco), 100 units $\cdot \mathrm{mL}^{-1}$ penicillin and $100 \mu \mathrm{g} \cdot \mathrm{mL}^{-1}$ streptomycin. Culture dishes were incubated at $37^{\circ} \mathrm{C}$ with $5 \% \mathrm{CO}_{2}$, and the medium was changed every 2 days. The cells were passaged when the cell fusion reached $80 \%$, as assessed under the microscope. The cells were then cultured with a-MEM supplemented with $10 \%$ FBS, $100 \mu \mathrm{g} \cdot \mathrm{mL}^{-1}$ streptomycin and 100 units per $\mathrm{ml}$ penicillin, 
Table 1. The primer sequences for qRT-PCR and siRNA

\begin{tabular}{ll}
\hline Gene & Primers sequences \\
\hline AFF1 & F:AAAGGCCAGCATGGATCAGAA \\
AFF2 & R:AAAGGCAGCATGGATCAGAA \\
& F:CTGACAGCGAATCTAATGAGGC \\
AFF3 & F:CATTGGTTGGATGATTGGAGGA \\
& R:AGTGCCTCTCTTACTCTGCTG \\
AFF4 & F:AAAGGCCAGCATGGATCAGAA \\
DLX5 & R:GTGATTTGGAGCGTTGATGTC \\
& F:CTACAACCGCGTCCCAAG \\
SP7 & R:CTACAACCGCGTCCCAAG \\
RUNX2 & F:CATCTGCCTGGCTCCTTG \\
B:CAGGGGACTGGAGCCATA \\
BGLAP & F:GTGCCTAGGCGCATTTCA \\
GAPDH & R:GCTCTTCTTACTGAGAGTGGAAGG \\
ALKBH1 & R:ACCTTTGCTGGACTCTGCAC \\
For siRNA & F:AGCCACATCGCTCAGACAC \\
AFF4 & R:GCCCAATACGACCAAATCC \\
& F:CGCGAGATGGGGAAGATGGCA \\
\hline qRT-PCR quantitative RT-PCR, siRNA & R:TCCAAGTCTCAGCTGTGAGG \\
\end{tabular}

and they were passaged every 4-5 days. Passage 3 cells were chosen for subsequent experiments.

Osteogenic induction

To induce osteogenic differentiation, the cells were grown in osteogenic medium containing $10 \mathrm{nmol} \cdot \mathrm{L}^{-1}$ dexamethasone (Sigma), $50 \mu \mathrm{mol} \cdot \mathrm{L}^{-1}$ ascorbic acid (Sigma) and $10 \mathrm{mmol} \cdot \mathrm{L}^{-1}$ $\beta$-glycerophosphate (Sigma) when the cells reached $50 \%-60 \%$ confluence.

\section{Gene knockdown}

For gene knockdown, we obtained a targeting control and an AFF4 siRNA from Sangon Biotech (Shanghai, China). The experimental protocols were performed according to the manufacturer's protocol. ${ }^{50}$ In brief, a transfection solution contained $50 \mathrm{nmol} \cdot \mathrm{L}^{-1}$ Lipofectamine $^{\mathrm{TM}}$ RNAiMAX in $200 \mu \mathrm{L}$ of Opti-MEM $^{\circledR}$ । Medium without serum was prepared and was incubated for $25 \mathrm{~min}$ at room temperature. When the cells reached $50 \%-70 \%$ confluence, they were diluted in complete growth medium without antibiotics, and the transfection solution with AFF4/control-siRNA was added and incubated with the cells for $10 \mathrm{~h}$ at $37^{\circ} \mathrm{C}$ in a $\mathrm{CO}_{2}$ incubator. The efficiency of the gene knockdown was assessed. The sequence of the AFF4-siRNA is recorded in Table 1.

\section{Gene overexpression}

For overexpression experiments, lentiviruses expressing empty vectors GFP and AFF4 were purchased from Genechem (Shanghai, China). The AFF4/control lentivirus infections were accomplished with Polybrene (Genechem). DFCs were cultured in 6-well culture plates to $30 \%-40 \%$ confluence, at which point they were infected with a lentivirus at a $\mathrm{MOI}=10$. After incubation for $72 \mathrm{~h}$ at $37^{\circ} \mathrm{C}$ in a $\mathrm{CO}_{2}$ incubator, the DFCs were selected by treatment with puromycin (Sigma) at a concentration of $2.5 \mu \mathrm{g} \cdot \mathrm{mL}^{-1}$ with continuous treatment until the cells were analysed for overexpression efficiency and then were used in subsequent experiments.
RNA isolation and qRT-PCR

With reference to the product directions, all RNA in this experiment was extracted with TRIzol reagent (Invitrogen), and cDNA was generated from RNA via a PrimeScript RT regent kit (Takara, Dalian, China) and gDNA eraser (Takara, Dalian, China).

qRT-PCR was performed with SYBR Premix Ex Taq (Takara, Dalian, China) and an ABI real-time PCR system (Applied Biosystems, Foster City, CA). The housekeeping gene GAPDH was regarded as a standard for normalizing expression. Using the $2^{-\Delta \Delta \mathrm{Ct}}$ method, the relative gene expression levels were demonstrated as the fold changes compared to the control. Thermocycling was performed as follows: step 1 (repeat 1 ) at $95^{\circ} \mathrm{C}$ for $30 \mathrm{~s}$, step 2 (repeat 40) at $95^{\circ} \mathrm{C}$ for $5 \mathrm{~s}$ and $60^{\circ} \mathrm{C}$ for $30-60 \mathrm{~s}$, and step 3 dissociation. The primer sequences for qRT-PCR are listed in Table 1.

\section{Western blot}

DFCs were lysed in 6-well plates with lysis buffer and protease inhibitor cocktail (Roche) on ice. To remove the cell debris, the cells were centrifuged for $15 \mathrm{~min}$ at $18000 \times g$ and $4^{\circ} \mathrm{C}$. The supernatants were obtained and heated for $5 \mathrm{~min}$ with sample buffer at $99^{\circ} \mathrm{C}$. The protein ( $15 \mu \mathrm{L}$ per sample) was loaded for analysis.

Protein lysates were transferred onto PVDF membranes by a transfer apparatus (Bio-Rad) ${ }_{1}^{51}$ and a-tubulin was chosen as a control marker. After immersion in blocking buffer ( $5 \%$ skim milk) for $1.5 \mathrm{~h}$, the membranes were incubated with rabbit anti-AFF4 (Abcam, 1:1 500), rabbit anti-ALKBH1 (Abcam, 1:1000), rabbit anti-a-tubulin (Sigma, 1:2 000), or rabbit anti-Flag (Sigma, 1:1 000) overnight at $4{ }^{\circ} \mathrm{C}$, which was followed by incubation with a secondary antibody for $1 \mathrm{~h}$ at room temperature. Then, the membranes were incubated with SuperSignal reagents (Pierce, Rockford, IL), and the antibody-antigen complexes were obtained. The semiquantification of protein levels was analysed by ImageJ software.

\section{ALP staining and activity}

DFCs underwent osteogenic induction in 24-well culture plates. After culturing with osteogenic differentiation medium for 7 days, the cells were collected for ALP staining. They were washed with PBS 3 times and then were fixed with paraformaldehyde..$^{52}$ Then, the cells were incubated with an ALP staining solution in the dark for 40 min; the solution was prepared from a BCIP/NBT Alkaline Phosphatase Color Development kit (Beyotime Biotechnology, Shanghai, China).

ALP activity was calculated with a Alkaline Phosphatase Assay kit (Beyotime Biotechnology, Shanghai, China) and BCA Protein Assay kit (Beyotime Biotechnology, Shanghai, China) in accordance with the manufacturer's protocols. Protein samples were diluted with $0.5 \mathrm{mmol} \cdot \mathrm{L}^{-1} p$-nitrophenyl phosphate from the Alkaline Phosphatase Assay kit. The BCA protein concentration was evaluated with absorbance measurements at $562 \mathrm{~nm}$ with a standard calcium curve.

\section{ARS staining}

After induction with osteogenic medium in 24-well culture plates for 3 weeks, DFCs were prepared for evaluation of mineralization ability. After washing with PBS 3 times, the DFCs were fixed with $4 \%$ paraformaldehyde for $25 \mathrm{~min}$ and then stained with alizarin red S solution (Solarbio Science \& Technology, Beijing, China) in the dark for $30 \mathrm{~min}$.

Quantitative analysis of calcium concentration

When alizarin red $S$ staining was finished, mineralized bone nodules were treated with $10 \%$ cetylpyridinium chloride for 15 min, which was dissolved in PBS. The quantitative analysis of calcium concentration was evaluated by collecting measurements at $562 \mathrm{~nm}$ with a standard calcium curve.

Chromatin immunoprecipitation (ChIP) assay

In accordance with the manufacturer's instructions, a ChIP assay was conducted via an EZ-Zyme Chromatin Prep kit (Millipore) and 
Magna Chip HiSens (Millipore) using either a normal rabbit IgG (Abcam) as a control or an antibody against AFF4 (Abcam) for the experimental group. First, the DNA-protein complexes were dissociated. Then, the pulled-down DNA as well as the input DNA were tested by qRT-PCR analysis, and the qRT-PCR primers were designed to identify the target promoter regions. The ChIP assay results are demonstrated in relation to the input DNA.

\section{Statistical analysis}

All data are presented as the mean \pm SE. Statistical differences were performed via unpaired two-tailed Student's $t$ test for comparisons between two groups or by one-way ANOVA followed by Tukey's post hoc test for multiple comparisons. A $P$ value of $<$ 0.05 was regarded as statistically significant.

\section{ACKNOWLEDGEMENTS}

This study was supported by a grant from the National Natural Science Foundation of China (NSFC, 81901040)

\section{AUTHOR CONTRIBUTIONS}

Q.X.: Contributed to design, data acquisition and interpretation, drafted and critically revised the manuscript. Y.Z.: Contributed to data acquisition and critically revised the manuscript. X.Q.: Contributed to data analysis and critically revised the manuscript. Y. C.: Contributed to data analysis and critically revised the manuscript. R.S.: Contributed to interpretation and critically revised the manuscript. R.X.: Contributed to data analysis and critically revised the manuscript. Q.Y.: Contributed to study design and critically revised the manuscript. C.Z.: Contributed to conception, design and critically revised the manuscript.

\section{ADDITIONAL INFORMATION}

Competing interests: The authors declare no competing interests.

\section{REFERENCES}

1. Chung, M., York, B. R. \& Michaud, D. S. Oral health and cancer. Curr. Oral. Health Rep. 6, 130-137 (2019).

2. Marchesan, J. T. et al. Role of inflammasomes in the pathogenesis of periodontal disease and therapeutics. Periodontol 2000 82, 93-114 (2020).

3. Carmagnola, D., Pellegrini, G., Dellavia, C., Rimondini, L. \& Varoni, E. Tissue engineering in periodontology: Biological mediators for periodontal regeneration. Int. J. Artif. Organs 42, 241-257 (2019).

4. Campanella, V. Dental stem cells: current research and future applications. Eur. J. Paediatr. Dent. 19, 257 (2018).

5. Liu, J. et al. Dental follicle cells rescue the regenerative capacity of periodontal ligament stem cells in an inflammatory microenvironment. PLoS ONE 9, e108752 (2014).

6. Cahill, D. R. \& Marks, S. C. Jr Tooth eruption: evidence for the central role of the dental follicle. J. Oral. Pathol. 9, 189-200 (1980).

7. Guo, W. et al. Dental follicle cells and treated dentin matrix scaffold for tissue engineering the tooth root. Biomaterials 33, 1291-1302 (2012).

8. Felthaus, O., Gosau, M., Ettl, T., Prantl, L. \& Morsczeck, C. Migration of human dental follicle cells in vitro. J. Periodontal Res. 49, 205-212 (2014).

9. Suzuki, A. et al. Daily low-intensity pulsed ultrasound-mediated osteogenic differentiation in rat osteoblasts. Acta Biochim Biophys. Sin. (Shanghai) 41, 108-115 (2009).

10. Zheng, C., Chen, J., Liu, S. \& Jin, Y. Stem cell-based bone and dental regeneration: a view of microenvironmental modulation. Int. J. Oral. Sci. 11, 23 (2019).

11. Fernandes, G. \& Yang, S. Application of platelet-rich plasma with stem cells in bone and periodontal tissue engineering. Bone Res. 4, 16036 (2016).

12. $\mathrm{Xu}$, L. et al. RNA polymerase II transcriptional fidelity control and its functional interplay with DNA modifications. Crit. Rev. Biochem. Mol. Biol. 50, 503-519 (2015).

13. Birkenheuer, C. H. \& Baines, J. D. RNA polymerase II promoter proximal pausing and release to elongation are key steps regulating herpes simplex virus 1 transcription. J. Virol. 94, e02035-19 (2019).

14. Peterlin, B. M. \& Price, D. H. Controlling the elongation phase of transcription with P-TEFb. Mol. Cell 23, 297-305 (2006).

15. Ehara, H. et al. Structural insight into nucleosome transcription by RNA polymerase II with elongation factors. Science 363, 744-747 (2019).

16. Chen, F. X. et al. PAF1, a Molecular Regulator of Promoter-Proximal Pausing by RNA Polymerase II. Cell 162, 1003-1015 (2015).
17. Knutson, B. A., Smith, M. L., Walker-Kopp, N. \& Xu, X. Super elongation complex contains a TFIIF-related subcomplex. Transcription 7, 133-140 (2016).

18. Lin, C. et al. Dynamic transcriptional events in embryonic stem cells mediated by the super elongation complex (SEC). Genes Dev. 25, 1486-1498 (2011).

19. Luo, Z. et al. The super elongation complex family of RNA polymerase II elongation factors: gene target specificity and transcriptional output. Mol. Cell Biol. 32, 2608-2617 (2012).

20. Melko, M. et al. Functional characterization of the AFF (AF4/FMR2) family of RNAbinding proteins: insights into the molecular pathology of FRAXE intellectual disability. Hum. Mol. Genet. 20, 1873-1885 (2011).

21. Schulze-Gahmen, U. et al. The AFF4 scaffold binds human P-TEFb adjacent to HIV Tat. Elife 2, e00327 (2013).

22. Kuzmina, A., Krasnopolsky, S. \& Taube, R. Super elongation complex promotes early HIV transcription and its function is modulated by P-TEFb. Transcription 8 , 133-149 (2017).

23. Zhou, C. C. et al. AFF1 and AFF4 differentially regulate the osteogenic differentiation of human MSCs. Bone Res. 5, 17044 (2017).

24. Zhou, C., Liu, Y., Li, X., Zou, J. \& Zou, S. DNA N(6)-methyladenine demethylase ALKBH1 enhances osteogenic differentiation of human MSCs. Bone Res. 4, 16033 (2016).

25. Asa'ad, F., Monje, A. \& Larsson, L. Role of epigenetics in alveolar bone resorption and regeneration around periodontal and peri-implant tissues. Eur. J. Oral. Sci. 127, 477-493 (2019).

26. Acil, Y. et al. Isolation, characterization and investigation of differentiation potential of human periodontal ligament cells and dental follicle progenitor cells and their response to BMP-7 in vitro. Odontology 104, 123-135 (2016).

27. Morsczeck, C. \& Reichert, T. E. Dental stem cells in tooth regeneration and repair in the future. Expert Opin. Biol. Ther. 18, 187-196 (2018).

28. Bok, J. S. et al. The Role of Human Umbilical Vein Endothelial Cells in Osteogenic Differentiation of Dental Follicle-Derived Stem Cells in In Vitro Co-cultures. Int. J. Med. Sci. 15, 1160-1170 (2018).

29. Allepuz-Fuster, P. et al. RNA polymerase II plays an active role in the formation of gene loops through the Rpb4 subunit. Nucleic Acids Res. 47, 8975-8987 (2019).

30. Chen, Y. et al. Prediction of RNA Polymerase II recruitment, elongation and stalling from histone modification data. BMC Genomics 12, 544 (2011).

31. Lu, H. et al. Gene target specificity of the Super Elongation Complex (SEC) family: how HIV-1 Tat employs selected SEC members to activate viral transcription. Nucleic Acids Res. 43, 5868-5879 (2015).

32. Chen, Y. \& Cramer, P. Structure of the super-elongation complex subunit AFF4 Cterminal homology domain reveals requirements for AFF homo- and heterodimerization. J. Biol. Chem. 294, 10663-10673 (2019).

33. Dahlberg, O., Shilkova, O., Tang, M., Holmqvist, P. H. \& Mannervik, M. P-TEFb, the super elongation complex and mediator regulate a subset of non-paused genes during early Drosophila embryo development. PLoS Genet. 11, e1004971 (2015).

34. Krasnopolsky, S. et al. Fused in sarcoma silences HIV gene transcription and maintains viral latency through suppressing AFF4 gene activation. Retrovirology 16, 16 (2019).

35. Christott, T. et al. Discovery of a selective Inhibitor for the YEATS domains of ENL/ AF9. SLAS Discov. 24, 133-141 (2019).

36. Monroe, S. C. et al. MLL-AF9 and MLL-ENL alter the dynamic association of transcriptional regulators with genes critical for leukemia. Exp. Hematol. 39, 77-86 (2011). e71-75.

37. Taki, T. et al. AF5q31, a newly identified AF4-related gene, is fused to MLL in infant acute lymphoblastic leukemia with ins(5;11)(q31;q13q23). Proc. Natl Acad. Sci. USA 96, 14535-14540 (1999).

38. Stevens, S. J. et al. A translocation in acute lymphoblastic leukemia that cytogenetically mimics the recurrent MLL-AFF1 translocation and fuses SEPT11 to MLL. Cancer Genet. Cytogenet 201, 48-51 (2010).

39. Yuva-Aydemir, Y., Almeida, S., Krishnan, G., Gendron, T. F. \& Gao, F. B. Transcription elongation factor AFF2/FMR2 regulates expression of expanded GGGGCC repeat-containing C9ORF72 allele in ALS/FTD. Nat. Commun. 10, 5466 (2019).

40. Metsu, S. et al. FRA2A is a CGG repeat expansion associated with silencing of AFF3. PLoS Genet. 10, e1004242 (2014).

41. Chou, S. et al. HIV-1 Tat recruits transcription elongation factors dispersed along a flexible AFF4 scaffold. Proc. Natl Acad. Sci. USA 110, E123-E131 (2013).

42. Deng, P. et al. AFF4 promotes tumorigenesis and tumor-initiation capacity of head and neck squamous cell carcinoma cells by regulating SOX2. Carcinogenesis 39, 937-947 (2018).

43. Markopoulos, G. S., Roupakia, E., Marcu, K. B. \& Kolettas, E. Epigenetic Regulation of Inflammatory Cytokine-Induced Epithelial-To-Mesenchymal Cell Transition and Cancer Stem Cell Generation. Cells 8, 1143 (2019).

44. Eslaminejad, M. B., Fani, N. \& Shahhoseini, M. Epigenetic regulation of osteogenic and chondrogenic differentiation of mesenchymal stem cells in culture. Cell J. 15, 1-10 (2013). 
AFF4 regulates osteogenic differentiation of human dental follicle cells Xiao et al.

45. Ougland, R. et al. Role of ALKBH1 in the core transcriptional network of embryonic stem cells. Cell Physiol. Biochem. 38, 173-184 (2016).

46. Muller, T. A., Struble, S. L., Meek, K. \& Hausinger, R. P. Characterization of human AlkB homolog 1 produced in mammalian cells and demonstration of mitochondrial dysfunction in ALKBH1-deficient cells. Biochem. Biophys. Res. Commun. 495, 98-103 (2018)

47. Deng, P., Chen, Q. M., Hong, C. \& Wang, C. Y. Histone methyltransferases and demethylases: regulators in balancing osteogenic and adipogenic differentiation of mesenchymal stem cells. Int. J. Oral. Sci. 7, 197-204 (2015).

48. Liang, Z. et al. DNA N(6)-Adenine Methylation in Arabidopsis thaliana. Dev. Cell 45, 406-416 e403 (2018).

49. Pan, Z. et al. Impaired placental trophoblast lineage differentiation in Alkbh1(-/-) mice. Dev. Dyn. 237, 316-327 (2008).

50. Guo, Y. C. et al. Ubiquitin-specific protease USP34 controls osteogenic differentiation and bone formation by regulating BMP2 signaling. EMBO J. 37, e99398 (2018).

51. Liu, W. et al. GDF11 decreases bone mass by stimulating osteoclastogenesis and inhibiting osteoblast differentiation. Nat. Commun. 7, 12794 (2016).
52. Wu, Y. et al. Mettl3-mediated m(6)A RNA methylation regulates the fate of bone marrow mesenchymal stem cells and osteoporosis. Nat. Commun. 9, 4772 (2018).

(i) Open Access This article is licensed under a Creative Commons Attribution 4.0 International License, which permits use, sharing adaptation, distribution and reproduction in any medium or format, as long as you give appropriate credit to the original author(s) and the source, provide a link to the Creative Commons license, and indicate if changes were made. The images or other third party material in this article are included in the article's Creative Commons license, unless indicated otherwise in a credit line to the material. If material is not included in the article's Creative Commons license and your intended use is not permitted by statutory regulation or exceeds the permitted use, you will need to obtain permission directly from the copyright holder. To view a copy of this license, visit http://creativecommons. org/licenses/by/4.0/.

(c) The Author(s) 2020 